\title{
Berühmte Schweizer Ärzte
}

\author{
Von Heinrich Buess und Marie-Louise Portmann
}

Im Jahre 1945 veröffentlichte Prof. H. Buess im Zusammenhang mit einer in der Universitätsbibliothek Basel gezeigten Ausstellung eine Broschüre, die den Titel trägt «Schweizer Ärzte als Forscher, Entdecker und Erfinder». Sie enthält 60 Lebensbilder bedeutender Schweizer Ärzte und wurde auch ins Französische und Italienische übersetzt.

In der Zeitschrift «Médecine et Hygiène», die in Genf erscheint und deren Chefredaktor Dr.P. Rentchnick ist, wird die Serie seit 23. November 1977 wieder abgedruckt unter dem Titel «Médecins suisses célèbres», vermehrt um 40 weitere Biographien, von denen vier in deutscher Fassung für den «Gesnerus» ausgewählt wurden. Für die Porträts verweise ich auf «Médecine et Hygiène».

M.-L.Portmann

\section{Johann Melchior Aepli}

geb.4. April 1744 in Dießenhofen

gest. 14. Januar 1813 in Konstanz

Immer wieder hat das Rheinstädtchen Dießenhofen der Medizin außergewöhnlich fähige Köpfe geschenkt. Schon Aeplis Vater Johann Konrad (1707-1787) war in seiner Vaterstadt dank glücklichen Operationen ein äußerst beliebter Arzt, so daß es nicht verwunderlich ist, wenn drei seiner Söhne den Beruf des Vaters wählten. Auch Johann Melchior, das Nesthäkchen der zehnköpfigen Familie, wuchs in den Beruf hinein. Eine seiner frühesten Beschäftigungen bestand darin, seinem Vater bei seiner Tätigkeit Handlangerdienste zu leisten. Schon früh lernte er Pflanzen zu sammeln für die Apotheke im Ordinationszimmer. Mit 16 Jahren war Aepli so weit, daß er im Wundarzt-Kollegium, in dem sich seit Wepfers Zeiten die Dießenhofener Ärzte und Chirurgen zu einer Art «Zunft» zusammengeschlossen hatten, als Lehrling eintreten konnte.

Das damalige medizinische Zentrum der Ostschweiz war Zürich. Dort trat Aepli beim Wundarzt Ludwig Wiser (1736-1816) zur Vervollständigung seiner Ausbildung ein. In theoretischer Beziehung besa $ß$ er an Heinrich Burkhard (1752-1799), dem späteren Lehrer an dem 1782 gegründeten medizinisch-chirurgischen Institut, einen warmherzigen Förderer. Auch der Naturforscher Johannes Gessner (1709-1790) nahm sich des strebsamen Thurgauers an. 
Als der ältere Bruder Andreas starb, beschloß der Vater, den Jüngsten àn seiner Stelle zum regelrechten Arzt auszubilden. Zu diesem Zweck wurde Aepli 1764 nach Tübingen geschickt, wo er durch die Vermittlung seines Studienfreundes Johann Friedrich Gmelin (1748-1804), der später Chemiker war, sich an dessen Vater, Friedrich Gmelin (1721-1768), anschloß. Damals erwarb sich Aepli die vorzüglichen Kenntnisse in der medizinischen Literatur, die ihn später zu seinen zahlreichen Publikationen befähigten. Nach der Disputation über ein pharmazeutisches Thema und seiner Promotion kehrte er im September 1765 nach Hause zurück.

Der junge Dießenhofener Arzt hatte bald ausgezeichnete Erfolge. Insbesondere eine Ruhr-Epidemie, die damals weite Teile der Schweiz heimsuchte, verlieh ihm großes Ansehen, da er nach dem Beispiel Tissots und Zimmermanns die veralteten Mittel abschaffte und die Diät in den Vordergrund stellte. Auch vom Cantharidenpflaster, das er mehrfach in Zeitschriftenbeiträgen empfahl, versprach er sich besondere Vorteile. Schon 1768 erhielt Aepli einen Ruf nach Rußland, lehnte ihn jedoch auf den Rat Gessners ab.

Als ursprünglicher Chirurg, d.h. als nicht akademische, sondern eher handwerksmäßige Medizinalperson, hatte Aepli für die Geburtshilfe eine besondere Vorliebe. Innert kurzer Zeit war er Meister seines Faches. Von allen Seiten wurde er zugezogen, besonders da die Hebammen ihrer Aufgabe in keiner Weise gewachsen waren. Aepli besaß eine geschickte und glückliche Hand und einen unvoreingenommenen Blick für das, was sich bewährte, und für das, was nicht der Natur entsprach. So wurde er nicht nur selbst auf ganz neue Wege geführt, sondern verstand es auch, die Helferin des Arztes, die Hebamme, nach seinen Grundsätzen auszubilden. Sein «Leitfaden für Hebammen» (1807) stellt ein Vermächtnis dar an die Ärzte, die selbst angehende Wehmütter zu erziehen hatten. In den Kantonen Thurgau und St. Gallen wurde Aeplis Werk für den Unterricht vorgeschrieben.

Den Höhepunkt von Aeplis Lebenswerk bildet seine bekannte «Abhandlung über die sichere Zurücklassung der Nachgeburt in bestimmten Fällen» (Zürich 1776). Er darf für sich die Ehre in Anspruch nehmen, als erster akademisch gebildeter Arzt und so nachdrücklich wie keiner vor ihm auf die Gefahren der aktiven Entfernung der Plazenta aufmerksam gemacht zu haben. Nachdem in einem Fall die Austreibung der Nachgeburt ohne jeglichen Schaden für die Mutter erst neun Tage nach der Geburt erfolgt war, ging Aepli dazu über, in allen Fällen die Ausstoßung den Kräften der Natur zu überlassen. Als Ausnahmen ließ er nur den fehlerhaften Sitz des Mutter- 
kuchens, heftige Blutungen und mangelhafte Austreibungskräfte bei sicherer Lösung der Plazenta von der Gebärmutterwand gelten.

Auch jüngeren Ärzten, wie zum Beispiel dem späteren helvetischen Politiker Paul Usteri (1768-1831), stand Aepli mit Rat und Tat bei. Usteri hatte sich als Student des Medicinisch-chirurgischen Institutes in Zürich an den Arzt in Dießenhofen gewandt, wobei sich eine Korrespondenz entwikkelte, die von 1786 bis 1789 dauerte. Aepli erhielt die Stelle des Hofrates und Leibarztes beim Fürsten von Hohenzollern und wurde in zahlreiche wissenschaftliche Gesellschaften aufgenommen.

Er hatte die Gedanken der französischen Aufklärer schon längst in sich aufgenommen, als die Stürme der Französischen Revolution bis in die äußerste Ostschweiz drangen. Als die Franzosen zu Beginn des Jahres 1798 in die Waadt einmarschierten, suchte Aepli dem neuen Geist auch in den alten Mauern seiner Vaterstadt Eingang zu verschaffen. Dabei stieß er allerdings auf Widerstand. Als Aepli sich gar den Bestrebungen der Helvetik mit Feuereifer anschloß und ihre Pläne in Dießenhofen zu verwirklichen suchte, kam es vollends zum Bruch zwischen dem vorwärtsdrängenden Arzt und seinen konservativen Mitbürgern. Das offenbar schon seit längerer Zeit gespannte Verhältnis endigte damit, daß Aepli nach Gottlieben übersiedelte. Dort fand er willigeres Gehör für die Ausführung seiner Pläne. Das Direktorium ernannte ihn zum Statthalter der helvetischen Regierung. In dieser Stellung blieb er während der Zeit der Helvetik. Durch die nach Stapfers Entwurf ausgearbeitete Thurgauische Verfassung wurde der neue Kanton in acht Bezirke oder Distrikte eingeteilt, die 32 Kreise umfaßten. Aeplis Verdienste in den Krisenjahren 1798-1803 wurden dadurch belohnt, daß er zum Distriktspräsidenten ernannt wurde. In der Zentralbehörde war er Vizepräsident des Sanitätsrates und Mitglied des Erziehungsrates.

In den letzten Lebensjahren zog sich der viel angefeindete Mann nach Konstanz zurück, wo er an den Folgen mehrerer Schlaganfälle starb. Er blieb ohne Nachkommen. Sein geistiger Erbe wurde jedoch sein Neffe Alexander Aepli (1767-1832), der Stammvater der St. Galler Linie, aus der mehrere bedeutende Juristen und Ärzte hervorgegangen sind. Als langjähriger Präsident der St.Gallischen Ärztekommission verpflanzte Alexander Aepli die fortschrittlichen Ideen seines Onkels auf neuen, fruchtbaren Boden. 


\section{Bibliographie (Auswahl)}

1. Jodocus Ehrharts Sammlungen von Beobachtungen zur Geburtshülfe 1773.

2. Abhandlung über die sichere Zurücklassung der Nachgeburt in bestimmten Fällen. Zürich 1776 .

3. Inoculations-Katechismus für das Landvolk. Rahns gemeinnütziges Magazin 1 (1782), S. $129 \mathrm{ff}$.

4. Ein brauchbares und bewährtes Mittel, den Kindermord zu verhüten. Rahns Magazin 2 (1783), S. 576.

5. Biographien berühmter Ärzte aus Dießenhofen. Rahns Archiv gemeinnütziger Kenntnisse 1, II (1787), S.435-577 (Fortsetzung in Rahns Wochenblatt physischen und medizinischen Inhalts 1792).

6. Von der Notwendigkeit einer Verbesserung des Medicinalwesens in der Schweiz. Winterthur 1788.

7. Leitfaden für Hebammen und ihre Lehrer, durch den Sanitätsrath des Cantons Thurgau zum Druck befördert. 1807.

\section{Biographie}

1. Alexander Aeplis Denkmal auf Johann Melchior Aepli, St.Gallen 1815. (Würdigung der einzelnen Schriften.)

2. Zusammenfassung des unter 1. genannten Lebensbildes durch Dekan Johann Conrad Ammann. Thurg. Neujahrsblatt 1828, S.17-24 (mit gutem Porträt).

3. Allgemeine Deutsche Biographie, Bd.1, Leipzig 1875, S. 130.

4. E.Moser, Dr.med.Johann Melchior Aepli aus Dießenhofen, ein thurgauischer Landarzt aus dem Ausgang des 18. Jahrhunderts. Gesnerus 3, Heft 4 (1946), S.174-192; 4, Heft 1 (1947), S. 43-59.

5. H.Buess, Geschichtlicher Überblick über die Entwicklung der Geburtshilfe. In: Th. Koller, Lehrbuch der Geburtshilfe, Bd. 1, Basel 1948.

6. Claude Henri Peter, Der Briefwechsel zwischen Paulus Usteri und Melchior Aepli. Diss. Zürich 1979. 
Rudolf Abraham Schiferli

geb. 30. September 1775 in Ammerswil (Aargau)

gest. 3. Juni 1837 in Bern

Die Familie Schiferli gehörte einem Thuner Geschlecht an, doch war der Vater Rudolf Abrahams Pfarrer im aargauischen Ammerswil, das damals zum Untertanenland Berns gehörte. Schiferli besuchte in Lenzburg die untere Mittelschule, wurde jedoch im 14. Lebensjahr zur Vorbereitung auf die Universität nach Bern geschickt. Der begabte Knabe sollte Theologe werden, doch nach dem Tode seines Vaters wählte er das Studium der Medizin. Da in Bern damals keine Möglichkeit bestand, «die oberen Fakultäten» zu besuchen, brachte der junge Student die ersten Semester in Jena zu, wo er sich an den besonders durch seine Vorlesungen über Makrobiotik berühmt gewordenen Christoph Wilhelm Hufeland (1762-1836) anschloß. Schiferli muß bereits zu Beginn seines Studiums über eine gute Vorbildung verfügt haben, denn schon nach einem Jahr (1796) wurde er auf Grund seiner Dissertation «De Cataracta» (über den grauen Star) zum Doktor der Medizin promoviert.

Der Begründer der wissenschaftlichen Hygiene, der Wiener Professor Johann Peter Frank (1745-1821), stand damals im Zenit seines Wirkens und übte großen Einfluß auf Schiferli aus, als dieser in Wien weilte. Von dort wandte er sich nach Paris, um die hochstehende französische Kriegschirurgie kennenzulernen, die in Larrey ihren berühmtesten Vertreter besaß. Schiferli machte sich in Paris bekannt, denn auf Grund einer Abhandlung über das System von John Brown (1735-1788), der die ganze Krankheitslehre auf dem Begriff der Erregbarkeit aufbaute, wurde er in die «Société de médecine de Paris» aufgenommen. In einigen kleineren Arbeiten, die er teilweise vor der Pariser Ärzteschaft vortrug, zeigte sich Schiferli als ausgezeichneter Beobachter und scharfer Denker. Um dieselbe Zeit ernannte ihn auch die «Göttingische Gesellschaft für Geburtshilfe» zu ihrem Mitglied.

Auch seine Heimat wurde auf den jungen Mitbürger aufmerksam. Seine Dissertation trug ihm zwei Medaillen aus Thun ein, und noch vor seiner Heimkehr wurde er in die «Helvetische Gesellschaft correspondierender Aerzte und Wundärzte» aufgenommen, die 1788 von dem Zürcher Johann Heinrich Rahn begründet worden war und die bis 1798 bestand. Schiferli wurde auch Mitglied der «Ökonomischen Gesellschaft» in Bern. Nach seiner Rückkehr nach Bern im Frühjahr 1798 zog er gegen das überhandnehmende 
Kurpfuschertum zu Felde. In der Aufklärung des Volkes durch Vorlesungen sah er das beste Gegenmittel.

Durch den Einmarsch der Franzosen kam es auch in Bern zu einem Umsturz. Schiferli, der in Paris reichlich republikanischen Geist geatmet hatte, stellte sich sofort rückhaltlos der neuen Regierung zur Verfügung. Durch seine führende Stellung in der Frage des medizinischen Unterrichts in Bern war Schiferli mit Albrecht Rengger (1764-1835), dem bedeutenden Staatsmann und Arzt, in nähere Berührung gekommen. Dieser hellsichtige Minister erkannte die Fähigkeiten Schiferlis und wußte sie der Helvetischen Republik nutzbar zu machen.

Während der ganzen Dauer der Helvetik (1798-1803) entfaltete Schiferli eine intensive Tätigkeit. Neben seiner kantonalen Professur am neugegründeten Medizinischen Institut in Bern wirkte er als eidgenössischer Sanitätsoffizier und Kriegschirurg. Schließlich hatte er auch noch eine private Praxis. Schon 1797 war er zum medizinischen Feldzeugwart ernannt worden, und im Februar 1799 wurde er in die Prüfungskommission für die sich meldenden Chirurgen berufen. Als die helvetische Armee gegen Österreich in Marsch gesetzt wurde, begleitete Schiferli die Truppe als Legionschirurg bereits im Majorsrang. Durch die kriegerischenVerhältnisse genötigt, erstrebte Schiferli eine allgemeine einheitliche Ordnung des Feldsanitätsdienstes. Während des Feldzugs des Jahres 1799 war Schiferli hauptsächlich als Chirurg tätig und damit beschäftigt, Lazarette herzurichten für die Tausende verwundeter Schweizer. In der helvetischen Armee bekleidete er nun den Rang eines Obersten. Als sich das Kriegsglück nach der Schlacht bei Zürich am 25./26. September 1799 wieder auf die Seite der Franzosen wandte, wurde die helvetische Armee überflüssig, und ihre Soldaten zerstoben, denn keiner wollte dem verhaßten Feind länger als nötig dienen. Was Bestand hatte, waren die Spitäler. Als einziges Lazarett blieben schließlich die engen Räume in der Insel in Bern, wo Schiferli zum Rechten sah. Auf sein Betreiben hin wurde am 12. Dezember 1799 das alte Johanniterschloß von Münchenbuchsee zum neuen Militärspital bestimmt und im Laufe der nächsten Monate eingerichtet.

Das Jahr 1801 brachte Schiferli auf den Höhepunkt seiner Wirksamkeit. Er amtierte als Präsident des medizinischen Instituts und seit dem 24. Dezember 1800 als «Inspektor der Gesundheitspflege», d. h. als erster eidgenössischer Oberfeldarzt. Es waren dies vorerst keine Einrichtungen von Dauer, doch ging von ihnen eine nachhaltige Wirkung für die Neugründungen späterer Jahre aus. Wie wenig auf dem Gebiet des Militärsanitätswesens 
vorgearbeitet war, beweist die Tatsache, daß Schiferli das Reglement für seine eigene Tätigkeit selber verfassen mußte. In seiner Privatpraxis nahm die Geburtshilfe einen großen Raum ein, sonst wäre der so vielseitig tätige Mediziner nicht in der Lage gewesen, schon 1806 ein «Handbuch der Entbindungskunst für Hebammen» herauszugeben, das ohne Zweifel nicht nur auf literarischem Wissen aufgebaut war.

Schiferlis Wirken als Leiter des helvetischen Militärsanitätswesens nahm ein rasches Ende, als es unter dem Einfluß reaktionärer Kräfte zum offenen Aufstand kam. Im «Stecklikrieg» (1802), der so genannt wird, weil ein großer Teil der Aufständischen nur mit Stöcken bewaffnet war, und der den deutsch-schweizerischen Teil der Auseinandersetzung zwischen dem konservativen Bund und dem helvetischen Vollziehungsrat darstellt, zog Schiferli in der Armee des Generals Andermatt als «Inspektor der Gesundheitspflege» mit ins Feld. In dem harmlosen Treffen bei Baden wurde Schiferli am 13. September 1802 mit vielen weiteren Offizieren gefangengesetzt. Auf allerlei Verdächtigungen hin, die Schiferli gegenüber von seiten seiner vielen Neider laut wurden, bat er am 9. Oktober 1802 um seine Entlassung als «Sanitätsinspektor».

Bei den Wahlen in die medizinische Fakultät der in der Mediation erweiterten Berner Akademie wurde Schiferli dann am 8. Mai 1805 als einer der wenigen Lehrer des «Medizinischen Instituts» zum Professor der Chirurgie und der Geburtshilfe sowie zum Dekan ernannt. Ein Jahr später finden wir ihn auch als Lehrer an der Hebammenschule. Dieser Auszeichnungen hatte sich Schiferli nicht nur als erfolgreicher Lehrer am «Medizinischen Institut», sondern auch als Vorkämpfer im Dienste der Volksgesundheit würdig erwiesen. Schon im Jahre 1800 hatte er sich in einer Schrift «Ein Wort über die Kuhpocken» für die neue Jennersche Impfmethode eingesetzt. Gestützt auf die guten Erfahrungen bei der Pockenvakzination anerbot sich der immer noch unternehmungsfreudige Arzt vier Jahre darauf, die Impfung der Kinder und Erwachsenen in Bern unentgeltlich vorzunehmen, damit dieses Geschäft den Händen der Quacksalber entrissen werde. Zum Dank dafür ernannte die Regierung Schiferli zum Oberimpfarzt des Kantons Bern. Er zählte auch zu den Gründern der «Medizinischchirurgischen Gesellschaft des Kantons Bern», die am 9. Oktober 1808 ins Leben gerufen wurde.

Da Schiferlis Gesundheitszustand später zu wünschen übrig ließ, mußte er auf die Praxis verzichten. Seine letzten zwanzig Jahre verbrachte er vor allem als Hofmeister der Anna Feodorovna. Sie war die Gattin des russischen 
Großfürsten Konstantin Pawlowitsch, der 1822 auf die Thronfolge zugunsten seines Bruders Alexander I. verzichtete. Die Fürstin wohnte auf der «Elfenau», einem Herrschaftsgut bei Bern, von wo Schiferli mit ausländischen Diplomaten, Fürsten und Gelehrten eine reiche Korrespondenz führte, die in der Berner Stadtbibliothek aufbewahrt wird und deren Herausgabe sich vielleicht lohnen würde. 1827 wurde Schiferli russischer Staatsrat, und von 1831 an war er Mitglied der Verfassungskommission der Stadt Bern und des Stadtrats. Er wurde mit hohen deutschen und russischen Orden ausgezeichnet.

\section{Bibliographie}

I. Selbständig erschienene Abhandlungen:

1. De cataracta. Diss. med. Jena 1796.

2. Theoretisch-praktische Abhandlung über den grauen Star. Jena und Leipzig 1797.

3. Analyse raisonnée du système de John Brown. Paris 1798.

4. Ein Wort über die Kuhpocken. Bern 1800.

5. Rede, gehalten bei der Eröffnung des Winterkurses in dem Medicinischen Institut zu Bern. Bern 1801.

6. Handbuch der Hebammenkunst zum Gebrauch bey Vorlesungen. Bern 1801.

7. Über den Einflu $\beta$ der Gemütsbewegungen auf Gesundheit und Lebensdauer. Bern 1808.

8. Erste und zweite Eröffnungsrede, gehalten in der Versammlung der medicinisch-chirurgischen Gesellschaft des Cantons Bern. Bern 1810 und 1811.

II. Von der helvetischen Regierung (Vollziehungsrat) genehmigte und teilweise in Zeitungen publizierte allgemeinverbindliche Reglemente von R. A. Schifferli:

1. Das erste «Reglement über die Stabsuniformen der helvetischen Armee» (Pt. 12-17 betr. San. Off.) vom 22. April 1799. Aktensammlung (vgl. unten), Bd.4, S. 280.

2. Festsetzung der Instruktion des Sanitäts-Inspektors für das helvetische Militär. Verfügt: Bern, 4. März 1801. Aktensammlung, Bd.5, S. 765 (Helvetisches Zentral-Archiv 751, 673, 675-681, 685-693, nicht gedruckt).

3. Reglement für die in den helvetischen Militärspitälern angestellten Wundärzte. Entwurf vom 8.Juni 1801. Genehmigt am 19. Juni (Helvetisches Zentral-Archiv 751, 713-722, nicht gedruckt). Akten-Sammlung zit., Bd.7, S. 69.

4. Verordnung betreffend die in den Militärspitälern aufzunehmenden Zöglinge. Erlassen am 11. März 1801. Aktensammlung, Bd.6, S. 775.

5. Reglement für die Feldchirurgen der Armee. Genehmigt 29.Juni 1801. (Helvetisches Zentral-Archiv 751, S.763-769; gleichlautend: Neuer Schweizer Republikaner 4, 1801, S.397-399). Zit. Akten-Sammlung, Bd.7, S.69.

6. Reglement für die in den helvetischen Militairspithälern angestellten Apotheker. Genehmigt am 1.August 1801 (Helvetisches Zentral-Archiv 751, S.731-735). Zit. in AktenSammlung, Bd.7, S. 69. 
7. Reglement für die Oekonomie-Officianten der Militärspitäler, Genehmigt am 3. September 1801. Der neue Schweizer Republikaner Jg.6, 10.September 1801, S.569-572 (Zit. Helvetisches Zentral-Archiv 751, S. 745). Zit. Akten-Sammlung, Bd.7, S. 70.

8. Polizeyreglement für die helvetischen Militairspithäler. Genehmigt am 16. September 1801 (Helvetisches Zentral-Archiv 751, S. 799-803). Zit. Akten-Sammlung, Bd.7, S. 70.

\section{Biographie}

1. Amtliche Aktensammlung aus der Zeit der helvetischen Republik. Herausgegeben von Johannes Strickler (10 Bände, Bern 1886-1910) und Alfred Rufer (Bd.11, Bern 1911).

2. M. Schiferli. Nekrolog in Schweiz. Zeitschr.f. Medizin, Chirurgie und Geburtshülfe 1 (1842), S. 92-96.

3. Ludwig Lauterburg, Rudolf Abraham Schiferli. Berner Taschenbuch 4 (1855), S.243-248.

4. O. Greyerz, Geschichte der Akademie in Bern. Berner Taschenbuch 20 (1871), S. 3-56.

5. R.von Fellenberg, Dr. Rudolf Abraham Schiferli 1775-1837. Gynaecologia helvetica 9 (1909), S.495-502 (mit bibliographischen Angaben, Porträt Schiferlis mit Autograph vor dem Titelblatt des Bandes).

6. Alfred Rufer, Schiferlis Bericht aus dem Jahre 1802 an den helvetischen Senat über die Organisation des Medizinalwesens der helvetischen Truppen. Schweiz. med. Wschr. 20 (1939), S.1311-1320.

7. Alville (Pseudonym für Mme. de W.), La vie en Suisse de S. A. I. la Grande-Duchesse Anna Feodorovna née Princesse de Saxe-Coburg-Saalfeld. Lausanne 1942 (Porträt u. a. Abbild.).

8. Ruth Jaussi, Das medizinische Institut in Bern (1797-1805). Bern 1944. (Schiferlis im Vordergrund stehende Leistung bei der Gründung des Instituts kommt deutlich zur Geltung.)

9. Heinrich Buess, Rudolf Abraham Schiferli, 1775-1837. Berner Zeitschr. f. Geschichte und Heimatkunde 1946, Heft 2.

10. M. Winzenried, Das schweizerische Militärsanitätswesen von der Mediation bis zum Sonderbundskrieg (Basler Veröff. z. Gesch. d. Medizin u. d. Biologie, Fasc. III), Basel 1954.

11. E.H.Ackerknecht und H.Buess, Kurze Geschichte der großen Schweizer Ärzte. Bern/Stuttgart/Wien 1975, S.45. 


\section{Adolf Haegler}

geb. 29. Juli 1830 in Lausen

gest. 12. August 1909 in Basel

Einem alten, weitverbreiteten Baselbieter Geschlecht entstammend, erblickte Haegler in der Mühle zu Lausen das Licht der Welt. Zunächst besuchte er die Bezirksschule im nahen Liestal. Da sich 1833 nach langen Kämpfen der Kanton Basel in zwei Halbkantone getrennt hatte, war es für Haegler zunächst nicht ratsam, die höheren Schulen in Basel zu besuchen. So ergab sich die Kantonsschule in Aarau als glücklicher Ausweg, besaß doch diese als Stätte der Vorbereitung auf die Hochschule einen guten Namen. Das Studium der Medizin, zu welchem sich Haegler entschloß, führte ihn zuerst nach Würzburg, dessen akademischer Lehrkörper durch Albert Koelliker aus Zürich 1847 einen erfreulichen Zuwachs erhalten hatte. Wie viele seiner Studiengenossen zog es auch Haegler dann zu den aufstrebenden Medizinschulen von Wien und Prag. Schließlich studierte er noch in Paris, wo ihm der augenärztliche Unterricht bei Desmarres den größten Eindruck gemacht zu haben scheint, wie das Vorwort zu seiner Dissertation «Über die Krankheiten des Thränenschlauchs» zeigt. Nachdem Haegler im Kanton Baselland das Staatsexamen bestanden hatte, erhielt er in Basel die medizinische Doktorwürde (1856).

Nach kurzer Wirksamkeit im heimatlichen Lausen ließ sich für Haegler in Fleurier im Val de Travers (Neuenburg) ein größeres Betätigungsfeld finden. Dort entwickelte sich die Praxis in kurzer Zeit zu höchster Blüte. Trotz der Wesensverwandtschaft, die ihn bei den Bewohnern des abgeschlossenen Juratales hatte heimisch werden lassen, sah sich Haegler im Interesse der Erziehung seiner Kinder veranlaßt, im Jahre 1863 nach Basel überzusiedeln, das sich nun von den Nachwirkungen der Kantonstrennung erholt hatte. Auch in den Basler Kreisen war der bereits erfahrene Arzt bald bestens eingeführt, und in der 1860 gegründeten medizinischen Gesellschaft war Haegler schon früh eines der tätigsten Mitglieder.

Auf den Vorschlag August Socins hin wurde Haegler 1865 als Stellvertreter des verstorbenen C. G. Jung an die medizinische Abteilung des Bürgerspitals berufen. Er versah die Stelle des Chefarztes in den schwersten Zeiten, die das 1842 eröffnete neue Spital erlebte. Enthält doch der Jahresbericht für das Jahr 1865 die gewaltige Zahl von 570 Typhuskranken, die unter den etwa 1700 Patienten bei weitem die größten Anforderungen an den Arzt und das übrige Personal stellten. Auf Grund der Angaben in den Verwaltungs- 
akten kann man Haegler das Zeugnis ausstellen, daß er seiner Aufgabe voll und ganz gewachsen war. Auch der im gleichen Jahr als Kliniker berufene Karl Liebermeister ist des Lobes voll über die hohen Fähigkeiten des interimistischen Spitalvorstehers. Am 25. September 1865 nahm Haegler seinen Rücktritt von dem ihm übertragenen Amt, weil er zur Überzeugung gekommen war, daß die gewissenhafte Besorgung des Spitals sich nicht auf die Dauer mit seiner bedeutenden Privatpraxis vereinbaren lasse. Seinem Votum ist es nicht zuletzt zu verdanken, daß man damals von der Ernennung eines nicht-klinischen Chefarztes absah.

In dem neuen Klinikvorsteher Karl Liebermeister (1833-1901) fand sich ein gleichdenkender Forscher, mit dem zusammen Haegler versuchte, der furchtbaren Seuche Einhalt zu gebieten. Haegler war einer der ersten, der zur Einsicht gelangte, daß die Krankheit durch Verschmutzung des Wassers mit typhösem Stuhl übertragen werde. Den Beweis dafür erbrachte Haegler, noch bevor man wußte, daß der Unterleibstyphus durch Bazillen erzeugt wird. Am 7.August 1872 brach in Lausen eine Epidemie aus, als deren Ausgangspunkt einige im Juni an Typhus erkrankte Personen eines oberhalb des Dorfes gelegenen Hofes ermittelt werden konnten. Statt die Exkremente der Kranken an vorgeschriebener Stelle zu vergraben, wie der Arzt den aufwartenden Leuten befohlen hatte, gelangten die Stuhlentleerungen in das am Hause vorbeifließende Bächlein und vermochten von dort aus das in der Nähe vorbeifließende Quellwasser zu verunreinigen. Sämtliche Familien in dem Dorf wiesen Typhuskranke auf, während in den Häusern mit eigenen Quellen primär keine Erkrankungen vorkamen. Als Beispiel für Haeglers klare Denkweise und logische Begabung mag von seinen Schlußfolgerungen das Wichtigste erwähnt werden: 1. Es ist ein strikter, sogar quantitativer Zusammenhang vorhanden zwischen dem Genuß des mit Typhusdejektionen vermischten Wassers und dem Ausbruch der Krankheit. 2. Zur Erzeugung des Abdominaltyphus bedarf es eines spezifischen, von Typhuskranken entstammenden Giftes. Haegler veröffentlichte seine Erkenntnisse 1873 im Deutschen Archiv für klinische Medizin. Sie trugen viel dazu bei, die Anschauung des Hygienikers Max von Pettankofer (1818-1901) zu erschüttern, der den Grundwasserschwankungen bei der Entstehung des Typhus entscheidende Bedeutung zuschrieb.

Dem tüchtigen Arzt brachte man auch von anderer Seite Vertrauen entgegen. Haegler wurde schon 1870 zum leitenden Arzt der Basler Lebensversicherungsgesellschaft ernannt. In dieser verantwortungsvollen Stellung legte er seine organisatorischen Fähigkeiten und seine wissenschaftlichen 
Interessen auch in mehreren gehaltvollen Schriften an den Tag. Fern aller einseitigen Spezialdisziplinen vertrat er hier allgemeingültige, ja zeitlose Ideen. Vor allem ist hier Haeglers Schrift «Über die Factoren der Widerstandskraft» (Basel 1896) durch ihre weitgehende Berücksichtigung der geistig-seelischen Komponente in der Beurteilung der Lebensaussichten auch über die Lebensversicherungsmedizin hinaus von hohem Wert.

In der zweiten Hälfte des letzten Jahrhunderts erlebte die Heilkunde dadurch eine gewaltige Bereicherung, daß neben der eigentlichen Krankenbehandlung auch für die Rekonvaleszenten eigene Erholungshäuser eingerichtet wurden. Nicht zuletzt dank dem unermüdlichen Eintreten Haeglers wurden auch in Basel für unbemittelte Rekonvaleszenten größere Summen gestiftet, und im Jahre 1888 erhielt das Bürgerspital ein Landhaus in Brüglingen zur Einrichtung einer Erholungsstation geschenkt. Ein weiteres Werk, dem Haegler seine ganze Kraft und seinen erfinderischen Geist widmete, war die Kinderheilstätte in Langenbruck, die im Mai 1884 eröffnet wurde. Auch an der Errichtung der Basler Heilstätte in Davos, einem Sanatorium für tuberkulöse Patienten (1897), war Haegler maßgebend beteiligt.

Bis in sein hohes Alter blieb der nimmermüde Arzt jung im Geiste, lernte mit den jüngeren Kollegen mit und nahm regen Anteil an der Entwicklung der medizinischen Wissenschaft. Als der Nestor der Basler Ärzte, dem die Stadt Basel das Ehrenbürgerrecht verliehen hatte, 1909 starb, betrauerten ihn zahllose Patienten und Freunde. Doch der Geist des großen Arztes und Menschenfreundes lebte weiter in seinem Sohn Carl Sebastian Haegler (1862-1914), der als Professor in Basel das ererbte Forschertalent auf die Chirurgie übertrug.

\section{Bibliographie}

1. Über die Krankheiten des Thränenschlauches und ihre Behandlung. Diss. med. Basel 1856.

2. Über die Aufgaben des Lebensversicherungs-Arztes. Anleitung für die Herren Vertrauensärzte der Basler Lebensversicherungsgesellschaft. Basel 1871.

3. Beiträge zur Entstehungsgeschichte des Typhus und zur Trinkwasserlehre. Dtsch. Arch.f. klin. Med. 11 (1873).

4. Der Sonntag vom Standpunkt der Gesundheitspflege und der Socialpolitik. Zwei öffentliche Vorträge. Basel 1878.

5. Der Sonntag als Grundlage von Gesundheit und Volkswohl. Basel o. J.

6. Über die Wirkung der Condurangorinde. Corr. Bl. f. Schweizer Aerzte 20 (1880), S.559, 491.

7. Die verschiedenartigen Bestrebungen unserer Zeit zur Fürsorge für Erholungsbedürftige. Zwei Vorträge. Basel 1891. 
8. Über Errichtung von Heilstätten für unbemittelte Lungenschwindsüchtige in der Schweiz. Corr. Bl.f. Schweizer Aerzte 23 (1893), S. 493.

9. Über die Behandlung der Lungenschwindsucht in Volksheilstätten. Öffentl. Vortrag (30. Okt. 1894). Basel 1894.

10. Über die Factoren der Widerstandskraft und die Vorhersage der Lebensdauer beim gesunden Menschen. Basel 1896.

11. Beruf und Beschäftigung. Auszug aus dem Handwörterbuch des gesamten Versicherungswesens. Herausgegeben von Eugen Baumgartner. O.J.

12. Im Kampfe gegen die Tuberkulose. Basel 1901.

\section{Biographie}

1. Ed.Hagenbach-Burckhardt, Adolf Haegler-Gutzwiller. Corr. Bl. f. Schweizer Aerzte 39 (1909), S. 865-868.

2. Rud.Dan.Oeri, Adolf Haegler-Gutzwiller. Basler Nachrichten, Jg.65 (1909), Nr.220 (14. Aug.).

3. Heinrich Buess, Adolf Haegler-Gutzwiller, ein Vorkämpfer für Gesundheit und Volkswohlfahrt. Sonntagsbl. Nationalztg., 7.0kt. 1945. 
Socin, der bedeutendste Basler Chirurg des 19. Jahrhunderts, war der Sohn eines Pfarrers in Vevey. Seine Mutter stammte aus Vevey, und so kam es, daß seine Muttersprache das Französische wurde und er nie deutsche Mundart sprach. Drei Tage nach Augusts Geburt starb der Vater, der aus einer alteingesessenen Basler Familie stammte. Die Mutter blieb zunächst mit ihren beiden Söhnen Karl und August in Vevey, bis sie 1849 nach Basel übersiedelte, wo die Brüder das Gymnasium absolvierten. August begann im Jahre 1854 das Medizinstudium in Basel. Da jedoch die dortige medizinische Fakultät zu jener Zeit nicht gerade in Blüte stand, zog er für das klinische Studium nach Würzburg, wo er so bedeutende Lehrer hatte wie Rudolf Virchow und Albert Koelliker. An seinem zwanzigsten Geburtstag promovierte Socin mit einer Dissertation über die chronische Bleivergiftung. Von 1857 bis 1858 besuchte er zu seiner Weiterbildung die Kliniken von Prag und Wien. Nach seinem Staatsexamen im Frühjahr 1859, das er in Basel ablegte, besuchte er Paris, wo er die bedeutenden französischen Kliniker kennenlernte und auch bereits die ersten größeren Arbeiten des russischen Kriegschirurgen Nikolai Iwanowitsch Pirogow las, welche einen entscheidenden Einfluß auf ihn ausübten.

Von Oktober 1859 an war Socin Assistenzarzt auf der chirurgischen Abteilung des Bürgerspitals Basel. Sein Vorgesetzter war Johann Jakob Mieg, dessen Nachfolger Socin 1862 wurde. Es wird überliefert, daß Socin in einem Fall von Fußverletzung seinem Chef eine Pirogowsche Amputation vorschlug.Dieser gab nach einigem Zögern sein Einverständnis. Das Ergebnis war so glänzend, daß Mieg den Ausspruch tat: «Unter einem solchen Assistenten kann ich nicht länger Oberarzt sein.» 1864 wurde Socin Ordinarius für Chirurgie in Basel. Mit dem Amtsantritt Socins war eine der Voraussetzungen erfüllt für den Wiederaufstieg der medizinischen Fakultät. Mehr und mehr Studenten kamen nach Basel, um von Socin unterrichtet zu werden. Er besaß ein ausgedehntes Wissen und kannte sich in der gesamten Fachliteratur gut aus.

Als Persönlichkeit wirkte er überzeugend und wußte durch seinen klaren, ungekünstelten Vortrag die Zuhörer zu fesseln. Er verstand es, sein medizinisches Wissen ex cathedra weiterzugeben, vernachlässigte aber dabei nicht den Unterricht der angehenden Ärzte am Krankenbett. 
Socin engagierte sich auch sehr für die Gründung der Medizinischen Gesellschaft, eine Vereinigung der Basler Ärzteschaft, die 1860 ins Leben gerufen wurde. Der sogenannte «Klinikenvertrag» von 1865 zwischen dem Kanton Basel-Stadt und der Stadtgemeinde Basel räumte den Chefärzten die Möglichkeit ein, sogenannte interessante Fälle von auswärts in Basel zu behandeln. So wurde das Einzugsgebiet der Universitätskliniken zugunsten der Ausbildung von Ärzten vergrößert. 1868 konnte die Bettenzahl durch den Ausbau des Spitals von ursprünglich 122 Betten verdoppelt werden.

Auf Socins Betreiben erfolgte 1865 die Herauslösung der geburtshilflichen Abteilung aus dem Verband der chirurgischen Klinik. Sie wurde Johann Jakob Bischoff unterstellt, einem früheren Assistenten Socins, mit dem dieser die Listersche Antiseptik in Basel einführte. Am 21. März 1868 operierte Socin zusammen mit seinem Assistenten Ludwig Georg Courvoisier erstmals einen Oberschenkelabszeß nach der neuen Methode, und am gleichen Tag legte er einem Amputierten am Oberarm einen antiseptischen Verband an, beides peinlich genau nach Listers Vorschriften. Damit war Socin nach Carl Thiersch in Leipzig der zweite auf dem Kontinent, dem das Verdienst zukommt, das Listersche Verfahren eingeführt zu haben. Im Jahre 1875 empfahl Thiersch einen neuen antiseptischen Verband, den Salicylwatte-Verband. Socin stellte genaue Indikationen auf, wann der «Lister» und wann der «Thiersch» zu verwenden sei. Er ging sogar so weit, daß er in gewissen Situationen eine Kombination der beiden Methoden als vorteilhaft betrachtete, womit er auch Erfolg hatte. Die Mortalität bei der Operation ging während seiner Amtszeit drastisch zurück, und auch die Heilungsdauer verkürzte sich.

Inzwischen war Socin international berühmt geworden. Immer wieder bekam er Berufungen an auswärtige Universitäten, so nach Bern, Marburg, Freiburg i.Br. und Würzburg. Socin schlug sie alle aus, denn die Stadt und die Universität Basel scheuten kein Mittel, um den geschickten Operateur für sich zu erhalten. Doch beschränkte sich sein Wirken nicht auf Basel. Er war auch auswärts tätig, und zwar als Kriegschirurg. Es war ihm ein Bedürfnis, sich in dieser Eigenschaft zum Wohl der Verletzten und Verwundeten einzusetzen. Im Jahr 1866 betätigte er sich während des ItalienischÖsterreichischen Krieges in den österreichischen Lazaretten in Verona und Ravenna. Als Anerkennung für seine Aufopferung in Oberitalien wurde Socin der «Hohe Orden der eisernen Krone» durch den österreichischen Kaiser verliehen.

Als dann 1870 der Deutsch-Französische Krieg ausbrach, wurde Socin 
vom Badischen Frauenverein als Leiter des in Bereitschaft gehaltenen Reservelazaretts am Karlsruher Bahnhof berufen. Während seiner kurzen Tätigkeit in Karlsruhe konnte Socin reichhaltige Erfahrungen sammeln, die es ihm nach seiner Rückkehr erlaubten, eine der vorzüglichsten kriegschirurgischen Publikationen zu erarbeiten, die zu jener Zeit erschienen. Das Buch trägt den Titel «Kriegschirurgische Erfahrungen, gesammelt in Karlsruhe 1870 und 1871». Das Material und die Unterlagen bildeten 643 Krankengeschichten, die er, wie er selber sagt, als seine Kriegstrophäe mit nach Basel nahm.

Als einer der ersten hatte Socin schon vor dem Deutsch-Französischen Krieg Schießversuche angestellt, um Klarheit zu gewinnen über die Wirkung von Gewehrprojektilen auf den menschlichen und tierischen Körper. Er wies nach, daß Schußwunden ohne irgendwelche Komplikation der sicheren Heilung zugeführt werden können. Falls eine Eiterung eintrat, rührte diese in der Mehrzahl der Fälle von Stoffresten von Hemd oder Uniform her, die jeweils in den Wunden aufgefunden wurden. Diese Tatsache belegte Socin anhand der viel rascheren Heilungstendenz von Schußwunden an unbedeckten Körperstellen wie Gesicht und Hals, im Gegensatz zu Schußwunden, die unter den Kleidern aufzusuchen waren.

Seine Erfahrungen stellte Socin auch dem schweizerischen Militärsanitätswesen zur Verfügung. Bei der Aus- und Weiterbildung der Sanitätsoffiziere sowie bei Revisionen des Militärsanitätswesens war Socins Mitarbeit sehr gefragt.

Der Basler Chirurg machte sich auch einen guten Namen als Urologe und als Hernienspezialist. Als im Jahr 1871 das «Handbuch der allgemeinen und speciellen Chirurgie» unter der Leitung der beiden Wiener Professoren Franz von Pitha und Theodor Billroth im Entstehen war, da wurde Socin angefragt, den Teil über die Krankheiten der Prostata zu verfassen. Er leistete der Aufforderung Folge. In seinen späteren Lebensjahren erhielt Socin abermals den Auftrag, für eine chirurgische Gesamtdarstellung das Kapitel über die Prostatakrankheiten zu verfassen. Es war wiederum Billroth, der Socin darum bat. Billroth begründete zusammen mit Albert Lücke das große Werk, das den Gesamttitel trug «Deutsche Chirurgie». Führende Chirurgen steuerten dazu ihre Beiträge bei. Dieses zweite Werk Socins erschien aber erst posthum 1902. Der Kollege und Mitarbeiter Socins, Emil Burckhardt, hat es vollendet. Socin war im Grunde nicht sehr glücklich darüber, daß er lediglich das Kapitel über die Prostatakrankheiten zur Bearbeitung erhalten hatte. Eine isolierte Betrachtung der Prostata schien 
ihm verfehlt. Er wies darauf hin, daß die Prostata nicht allein eine zum Genitalapparat gehörende Drüse enthalte, sondern daß sie einen integrierenden Bestandteil des gesamten Urogenitalsystems bilde. Socin stellte vor allem den engen anatomischen und physiologischen Zusammenhang der Prostata mit der Harnblase in den Vordergrund.

Socin war auch ein Pionier in der Radikaloperation der Hernien. Mit der Einführung der Antiseptik hatten sich auch hier neue Möglichkeiten eröffnet. Im Jahre 1888 hielt Socin vor dem «Congrès français de chirurgie» in Paris einen bemerkenswerten Vortrag «Sur les résultats définitifs de la cure radicale de la hernie». Anhand seiner 160 ausgeführten Radikaloperationen von Inguinal- und Cruralhernien bewies Socin mittels seiner Verlaufsbeobachtungen und Nachuntersuchungen, daß eine völlige Heilung nicht nur möglich ist, sondern daß in bestimmten Fällen eine Heilung ausschließlich von der Radikaloperation erwartet werden darf.

Socin war mitbeteiligt an der Gründung der Deutschen Gesellschaft für Chirurgie, die 1872 in Berlin erfolgte. Auch bei der Entstehung des französischen «Congrès de chirurgie» wirkte er mit. So kam es, daß Socin immer wieder mit den führenden Fachkollegen aus aller Welt zusammentraf und dabei viele Freunde gewann. Er beteiligte sich rege an den Diskussionen, und seine Meinung besaß bei den Fachkollegen großes Gewicht.

Mit Vorliebe verbrachte er seine Mußestunden auf der Jagd. Noch 14 Tage vor seinem Tod ging er mit seinen Freunden dem edlen Weidwerk nach. Jedoch am Tage nach der letzten Jagd mußte er sich niederlegen. Gefaßt und ruhig sah er seinem Tod entgegen und traf seine letzten Verfügungen. Mit klarem Geist nahm er Abschied von seinen Freunden und Schülern und starb am Sonntag, den 22. Januar 1899. Es fand eine große Abdankungsfeier statt, wo Angehörige und Freunde sowie Leute aus allen Schichten der Bevölkerung dem bedeutenden Chirurgen und liebenswürdigen Menschen die letzte Ehre erwiesen.

\section{Bibliographie}

1. Die Krankheiten der Prostata. In: Handbuch der allgemeinen und speciellen Chirurgie, redigiert von F.v.Pitha und Th.Billroth, Dritter Band, Zweite Abteilung, Stuttgart 1871-1875.

2. Kriegschirurgische Erfahrungen gesammelt in Carlsruhe 1870 und 1871. Leipzig 1872.

3. Ein Fall von akuter, traumatischer Kniegelenkseiterung mit Beibehaltung der Funktion geheilt. Corr. bl.f. Schweizer Aerzte, 1874, S. 353-358.

4. Zur Behandlung der Kopf-Verletzungen. Corr. bl.f. Schweizer Aerzte 1876, S. 706.

5. Zur Magenchirurgie. Corr. bl.f. Schweizer Aerzte 1884, S.513-517.

6. Zur Interpretation des Haftpflichtgesetzes. Corr. bl.f. Schweizer Aerzte 1887, S. 545-554. 
7. Sur les résultats définitifs de la cure radicale de la hernie. Extrait du Congrès Français de Chirurgie. Paris 1888.

8. Das Koch'sche Heilverfahren auf der chirurgischen Klinik. Corr. bl. f. Schweizer Aerzte 1891, Beilage No.1.

9. Theodor Billroth. Corr. bl.f. Schweizer Aerzte 1894, S. 129-136.

10. Über die Bedeutung der Kastration für die Behandlung der Prostatahypertrophie. Corr. bl.f. Schweizer Aerzte 1896, S.529-537.

11. Die Verletzungen und Krankheiten der Prostata. In: Deutsche Chirurgie, begründet von Th. Billroth und A. Lücke. Lief. 53, Stuttgart 1902.

\section{Biographie}

1. Emil Burckhardt, August Socin. Nachruf. Basler Nachrichten, 25. Januar 1899.

2. Charles Byse, Biographische Notizen. In: Zur Erinnerung an Herrn Professor August Socin. Basel 1899.

3. Ludwig Georg Courvoisier, Prof.Dr.August Socin. Corr. bl. f. Schweizer Aerzte 1899, S. 257-264.

4. Carl Sebastian Haegler, August Socin. Münchener med. Wschr. (1899), No. 10.

5. Wilhelm Von der Mühll-Merian, Nachruf an Herrn Prof.Dr. Aug. Socin. In: Worte der Erinnerung an Prof. Dr. August Socin, hrsg. von Rudolf Vischer-Burckhardt. Basel 1899.

6. Eduard His, Basler Gelehrte des 19. Jahrhunderts. Basel 1941.

7. Heinrich Buess, August Socin zum 50. Todestag. Basler Nachrichten, 22./23. Januar 1949.

8. Gwer Reichen, Die chirurgische Abteilung des Bürgerspitals Basel zur Zeit der Antiseptik. Ein Beitrag zur Geschichte der Basler chirurgischen Klinik (Veröffentlichungen der Schweizerischen Gesellschaft für Geschichte der Medizin und der Naturwissenschaften XVIII), Aarau 1949.

9. Rudolf Nissen, August Socin. In: Professoren der Universität Basel aus fünf Jahrhunderten, hrsg. von Andreas Staehelin, S. 192-193. Basel 1960.

10. Huldrych M. Koelbing, Das Bürgerspital als Universitätsspital 1865-1965. In: 700 Jahre Bürgerspital Basel 1265-1965. Hrsg. Bürgerspital Basel. Basel 1965.

11. Erwin H. Ackerknecht und Heinrich Buess, Kurze Geschichte der großen Schweizer Ärzte. Bern/Stuttgart/Wien 1975, S. 64.

12. Samuel Meier, August Socin (1837-1899). Diss. med. Basel 1979.

\section{Summary}

Four short biographies of Swiss physicians are presented in this paper, namely J.M. Aepli (1744-1813, physician and politician), R.A.Schiferli (1775-1837, surgeon and obstetrician), A. Haegler (1830-1909, medical director of the Basle hospitals), and A. Socin (1837-1899, the famous Swiss surgeon).

Prof. Dr. med. Heinrich Buess und Dr. Marie-Louise Portmann

Medizinhistorisches Institut

Klingelbergstraße 23

CH-4031 Basel 